\title{
Die distale Radiusfraktur Konservative Behandlung: Indikation und Durchführung
}

\author{
Wolfgang Schneiders, Stefan Rammelt, Hans Zwipp
}

\section{Zusammenfassung}

Die distale Radiusfraktur ist mit einem Anteil von 10-25\% aller Frakturen der häufigste Knochenbruch des Menschen. Durch die Anwendung von differenzierten Behandlungsschemata konnten die Behandlungsergebnisse der distalen Radiusfraktur deutlich verbessert werden. Die konservative Frakturbehandlung hat hierbei ihren Stellenwert bei nicht dislozierten und den gering dislozierten extraartikulären Frakturen sowie bei der Behandlung distaler Radiusfrakturen bei betagten Patienten. In diesen Fällen führt die konservative Behandlung zu überwiegend guten bis sehr guten Behandlungsergebnissen. In der Gruppe der älteren Patienten mit geringerem Funktionsanspruch muss darauf hingewiesen werden, dass eine gewisse Fehlstellung verbleiben kann, die sich in den meisten Fällen funktionell wenig bemerkbar macht.

\section{Distal Radius Fractures Indications and Techniques of Conservative Therapy}

A fracture of the distal radius is the most common fracture in humans (10-25\% of all fractures). By using a sophisticated and individualised treatment plan the outcome of these fractures has been optimised during the last 20 years. Conservative treatment is indicated in non-displaced intraarticular fractures, non- or only minimally displaced extraarticular fractures and in elderly patients with low functional demands. In these cases conservative treatment leads to good results in most cases. In the group of elderly patients an unsatisfactory radiographic outcome does not necessarily translate into an unsatisfactory functional outcome.

\section{Einleitung}

Die distale Radiusfraktur ist mit einem Anteil von 10-25\% aller Frakturen der häufigste Knochenbruch des Menschen. Die Inzidenz beträgt derzeit 2-3 pro 1000 Einwohner pro Jahr mit steigender Tendenz. Aus der Epidemiologie lassen sich generell 2 Ursachengruppen unterscheiden [6,7]. Die (häufigere) ältere Generation erleidet typischerweise im Rahmen eines einfachen Sturzes eine distale Radiusfraktur. In dieser Gruppe findet sich ein deutliches Überwiegen von weiblichen Patienten. Durch die demografisch bedingte Veränderung der $\mathrm{Al}$ tersstruktur in der Bevölkerung ist in

OP-JOURNAL 2012; 28: 240-242

(c) Georg Thieme Verlag KG Stuttgart · New York DOI http://dx.doi.org/10.1055/s-0032-1327994 den kommenden Jahrzehnten ein deutlich höherer Anteil geriatrischer Patienten zu erwarten. Neben dem Sturzereignis ist die verminderte mechanische und strukturelle Knochenqualität ein wesentlicher Faktor, der die Frakturentstehung begünstigt. Der Zusammenhang zwischen distaler Radiusfraktur und Osteoporose konnte inzwischen in mehreren Studien belegt werden. So wiesen Owen et al. bei 30\% von knapp 1800 Patienten über 50 Jahren mit einer distalen Radiusfraktur eine zuvor nicht diagnostizierte Osteoporose nach [6]. Somit sollte die distale Radiusfraktur nach einem Niedrigenergietrauma immer als Warnsignal für die Osteoporose gesehen werden.

Die 2. Gruppe ist gekennzeichnet durch jüngere Patienten, die die distale Radiusfraktur im Rahmen eines sog. Mittel- oder Hochenergietraumas erleiden. In dieser Gruppe findet sich ein Überwiegen des männlichen Geschlechts.

Die konservative Frakturbehandlung hat ihren Stellenwert bei gering dislozierten extraartikulären und nicht dislozierten Frakturen sowie bei der Behandlung distaler Radiusfrakturen bei betagten Patienten.

\section{Hauptteil}

Die Spannbreite der operativen Versorgung der distalen Radiusfraktur ist groß. Sie reicht von der konservativen Behandlung über die Kirschner-Draht-Osteosynthese, den Verriegelungsnagel für den distalen Radius, die winkelstabile und nicht winkelstabile Plattenosteosynthese bis zur arthroskopisch gestützten Versorgung. Bis Mitte der Achtzigerjahre des letzten Jahrhunderts war die Behandlung der distalen Radiusfraktur eine Domäne der konservativen Behandlung. Jedoch führte die zu diesem Zeitpunkt nahezu ausschließlich konservative Therapie in bis $\mathrm{zu} 30 \% \mathrm{zu}$ unbefriedigenden Behandlungsergebnissen. Durch die Anwendung differenzierter Behandlungsschemata in den letzten 20 Jahren konnten die Behandlungsergebnisse zunehmend verbessert werden. Die Grundlage dieser Behandlungsschemata sind die AO-Klassifikation, Begleiterkrankungen, biologisches Alter, Allgemeinzustand des Patienten (körperlich und mental) sowie der Funktionsanspruch des Patienten und dessen Bedürfnisse.

Als etablierte Indikationen für die konservative Behandlung gelten

- stabile extraartikuläre Frakturen,

- nicht oder gering dislozierte, intraartikuläre Frakturen,

- lokale oder allgemeine Kontraindikationen gegen die Operation.

Relative Indikationen für die konservative Behandlung sind primär reponierbare und retinierbare Frakturen mit 


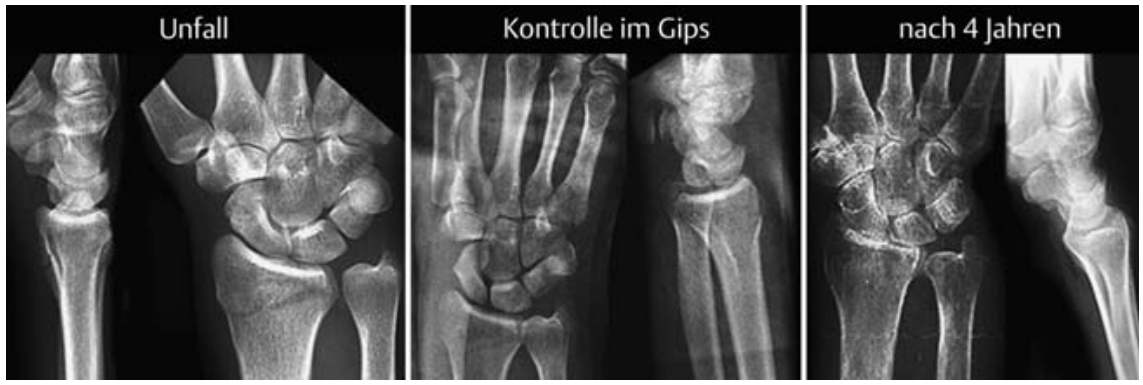

Abb. 1 52-jähriger Patient, der sich bei einfachem Sturz eine gering dislozierte Radiusfraktur (A2) zugezogen hatte. Nach der Reposition erfolgte die Gipsanlage und radiologische Kontrolle. Nach 4 Jahren zeigte sich im Rahmen einer Nachuntersuchunng ein sehr gutes radiologisches, subjektives und funktionelles Ergebnis.

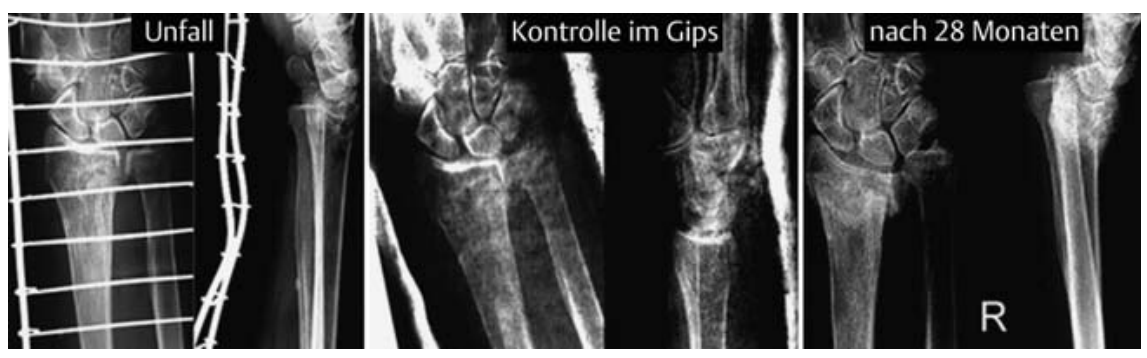

Abb.2 62-jähriger Patient mit einer deutlich nach dorsal abgekippten distalen Radiusfraktur (A3). Der Patient lehnte die operative Versorgung ab. Das Repositionsergebnis zeigt noch ein sehr gutes radiololgisches Ergebnis. Dieses kann jedoch durch die alleinige Retention im Gips nicht gehalten werden. Nach 28 Monaten zeigt sich bei einer Nachuntersuchung die sekundäre Redislokation mit erheblichem Ulnarvorschub und dadurch bedingtem eingeschränktem funktionellem Ergebis.

Instabilitätskriterien. Nach Jupiter [4] werden hierbei folgende Instabilitätskriterien definiert: dorsale Trümmerzone, Mehrfragmentfraktur, Verlust der radialen Länge $>2 \mathrm{~mm}$, Dorsalabkippung $>20^{\circ}$, assoziierte Ulnafraktur, radioulnare Instabilität.

\section{Technik}

Nicht dislozierte Frakturen können ohne jegliches Repositionsmanöver zunächst in einer Unterarmgipslonguette und nach Abschwellung in einem zirkulierten Unterarmgips für 4 Wochen ruhig gestellt werden. Nach dorsal dislozierte Frakturen sollten initial nach Überprüfung der Motorik, Sensibilität und Durchblutung reponiert werden. Als Anästhesieformen stehen mehrere Verfahren zur Auswahl. Neben der Bruchspaltanästhesie kommen in seltenen Fällen auch regionale Leitungsanästhesien zur Anwendung. Die Reposition erfolgt durch Zug und Gegenzug entweder mit einem sog. „Mädchenfänger“ oder manuell. Bei der Repositionstechnik mit dem „Mädchenfänger“ liegt der Patient auf dem Rücken, der Oberarm ist um $90^{\circ}$ abduziert und der Unterarm befindet sich in Neutralposition. Durch
3 Schlaufen werden der Daumen, der 2. und 4. Finger fixiert. Die Extension wirkt primär radial, wobei die Fixierung des 2 . und 4. Fingers einen Rotationsschutz darstellt. Die Extensionsdauer sollte bei einem Zuggewicht zwischen 3 und $5 \mathrm{~kg}$ bei 10 Minuten liegen. Additiv sollte nach 5 Minuten ein manuelles Repositionsmanöver mit Ulnarduktion und Flexion im Handgelenk bei gleichzeitigem Druck auf das distale Fragment erfolgen. Bei der manuellen Reposition nach Charnley werden die Frakturflächen der beiden Fragment als 2 Zahnräder gesehen [3]. Durch die Dorsalextension und Distraktion wird zunächst das falsche Ineinandergleiten der 2 Zahnräder ausgeglichen, sodass dann durch ein Flexionsmanöver durch Druck von dorsal auf das periphere Fragment die Reposition erfolgt. Prinzipiell sollte ein Repositionsmanöver nicht brüsk oder wiederholt erfolgen, da hierdurch die Ausbildung eines CRPS begünstigt werden kann.

Nach der Reposition erfolgt die Retention in einer dorsalen Unterarmgipsschiene oder einem gespaltenen Unterarmgips. An der Ulnaseite soll diese bis an die Beugeseite der ulnaren Handkante, an der Radialseite über den 1. Mittel- handknochen bis auf den Daumenballen reichen. Die durchschnittliche knöcherne Heilungszeit der distalen Radiusfraktur wird mit 4-6 Wochen angegeben. Röntgenkontrollen werden unmittelbar nach dem Repositionsmanöver am 4., 7. und 14. sowie 28. Tag empfohlen. Die Zirkulation des Gipses - idealerweise unter leichter Distraktion im „Mädchenfänger" - kann in Abhängigkeit vom Weichteilbefund zwischen dem 4. und 7. Tag erfolgen. Zusätzlich sollte am 1. Tag nach dem Repositionsmanöver eine Gipskontrolle mit Überprüfung der Motorik, Sensibilität und Durchblutung der Finger erfolgen.

Prinzipiell sollte ein Repositionsmanöver nicht brüsk oder wiederholt erfolgen, da hierdurch die Ausbildung eines CRPS begünstigt werden kann.

Ergebnisse und Probleme der konservativen Behandlung

Die Behandlungsergebnisse der konservativen Behandlung sind unterschiedlich. In den Fällen, in denen die konservative Behandlung im Rahmen eines differenzierten Behandlungsschemas angewendet wurde, führt die konservative Behandlung in weit über $90 \%$ der Fälle $\mathrm{zu}$ guten bis sehr guten Behandlungsergebnissen [7]. In diesen Fällen handelt es sich gewissermaßen um die einfachsten Frakturformen des distalen Radius, die A2- und die nicht dislozierten Gelenkfrakturen. Jedoch zeigten sich in Studien, bei denen die Indikation zur konservativen Behandlung weiter gefasst wurde, auch schlechtere Behandlungsergebnisse. In diesen Studien liegt der Anteil von guten bis sehr guten Behandlungsergebnissen zumeist zwischen 65 und 75\%. Als Hauptproblem wird in diesen Arbeiten die Rate an sekundären Korrekturverlusten gesehen. So berichteten Altissimi et al. über $71 \%$ an sekundären Dorsalabkippungen und $47 \%$ an sekundären Sinterungen bei 100 konservativ behandelten distalen Radiusfrakturen [1]. Die Korrelation zwischen klinischem und radiologischem Ergebnis bei der distalen Radiusfraktur wird in der Literatur kontrovers diskutiert. Unserer Meinung nach gibt es 2 Ursachen für die unterschiedliche Wertung der radiologischen Ergebnisse hinsichtlich der Bedeutung für das funktionelle und subjektive Ergebnis. Zum einen werden unterschiedliche Populationen verglichen. Die Studien, welche keinen oder nur einen geringen $\mathrm{Zu}$ sammenhang zwischen radiologischem 
und funktionellem bzw. subjektivem Ergebnis sehen, beziehen sich zumeist auf die Behandlung von Patienten im fortgeschrittenen Alter mit einem oft geringeren Anspruch an die Handgelenksfunktion. Zum anderen fehlt eine differenzierte Betrachtung der radiologischen Ergebnisse. Trumble et al. zeigen in ihrer Nachuntersuchung von 49 Patienten, dass der Zustand der Gelenkflächen und eine Verkürzung des Radius signifikant mit dem funktionellen Ergebnis korrelierten [10]. Die palmare Inklination hatte hingegen keinen Einfluss auf das funktionelle Ergebnis. Auch Kreder et al. [5] bestätigten, dass verbliebene Gelenkstufen zu einer signifikanten Verschlechterung des Behandlungsergebnisses führen. Eigene Ergebnisse bestätigen diese Untersuchungen [7]. Biomechanische Studien haben zusätzlich gezeigt, dass eine vermehrte Dorsalabkippung u.a. zu einer Inkongruenz des distalen Radioulnargelenks führt.

Zusammenfassend sollte somit aufgrund der hohen Redislokationsrate die Indikation für die konservative Behandlung insbesondere bei aktiven Patienten nur bei sog. stabilen extraartikulären Frakturen und bei nicht oder nur gering dislozierten, intraartikulären Frakturen gesehen werden.

Hauptproblem der konservativen Behandlung ist die Rate an sekundären Korrekturverlusten.

\section{Konservative Behandlung beim geriatrischen Patienten}

Bei betagten Patienten ist die konservative Behandlung der distalen Radiusfraktur als Behandlungsalternative auch bei instabilen Frakturen immer in Erwägung zu ziehen [2,9]. In mehreren Studien ist nachgewiesen, dass auch bei radiologisch nicht perfektem Behandlungsergebnis die überwiegende Anzahl in dieser Patientengruppe subjektiv mit dem Ausheilungsergebnis zufrieden ist und funktionell, insbesondere wenn die nicht dominante Seite betroffen ist, bei den Alltagsbewegungen nicht wesentlich eingeschränkt ist. Die Ausbildung einer posttraumatischen Arthrose ist in dieser Patientengruppe bei auch deutlich geringerer Belastung der Handgelenke nur bedingt zu erwarten. Nur bei ausgeprägten Fehlstellungen oder evtl. Medianusaffektion sollte die offene Reposition und Retention mit einer winkelstabilen Plattenosteosynthese erfolgen. Young et al. [9] zeigten bei 25 Patienten mit einem Altersdurchschnitt von 72 Jahren 3 Jahre nach konservativer Behandlung, dass kein Zusammenhang zwischen dem funktionellen und radiologischen Ergebnis bestand. Arora et al. [2] konnten in ihrer Studie mit mehr als 130 Patienten von über 70 Jahren sogar zeigen, dass es keinen Unterschied der funktionellen oder subjektiven Behandlungsergebnisse zwischen konservativ behandelten und mit palmarer winkelstabiler Platte versorgten Patienten nach 1 Jahr gab. Auch minimalinvasive Verfahren wie die Kirschner-Draht-Osteosynthese sollten bei diesen Patienten aufgrund der häufigen vorzeitigen Lockerung der Drähte bei reduzierter Knochenqualität nicht das Verfahren der Wahl sein. Insbesondere bei zusätzlichem Vorliegen einer Demenzerkrankung ist die Kirschner-Draht-Osteosynthese kontraindiziert, da hier immer wieder Fälle beschrieben sind, bei denen Patienten an den Kirschner-Drähten manipulierten mit Ausbildung revisionspflichtiger Osteitiden [8].

\section{Schlussfolgerung}

Die konservative Frakturbehandlung hat ihren Stellenwert bei nicht dislozierten und den gering dislozierten extraartikulären Frakturen sowie bei der Behandlung distaler Radiusfrakturen bei betagten Patienten. In diesen Fällen führt die konservative Behandlung zu überwiegend guten bis sehr guten Behandlungsergebnissen. In der Gruppe der betagten Patienten muss darauf hingewiesen werden, dass eine gewisse Fehlstellung verbleiben kann, die sich meistens funktionell wenig bemerkbar macht.

\section{Literatur}

${ }^{1}$ Altissimi M, Mancini GB, Azzarà A et al. Early and late displacement of fractures of the distal radius. The prediction of instability. Int Orthop 1994; 18: 61-65

2 Arora R, Gabl M, Gschwentner M et al. A comparative study of clinical and radiologic outcomes of unstable colles type distal radius fractures in patients older than 70 years: nonoperative treatment versus volar locking plating. J Orthop Trauma 2009; 23: 237-242

3 Charnley J. Die konservative Therapie der Extremitätenfrakturen. Berlin, Heidelberg, New York: Springer; 1968

${ }^{4}$ Jupiter JB. Fractures of the distal end of the radius. J Bone Joint Surg [Am] 1991; 73: 461469

${ }^{5}$ Kreder HJ, Handel DP, Agel J et al. Indirect reduction and percutaneous fixation versus open reduction and internal fixation for displaced intraarticular fractures of the distal radius. J Bone Joint Surg [Br] 2005; 87: 829836

${ }^{6}$ Owen RA, Melton III LJ, Johnson KA et al. Incidence of Colles' fracture in a North American community. Am J Public Health 1982; 72: 605-607

7 Schneiders W, Biewener A, Rammelt S et al. Die distale Radiusfraktur, Korrelation zwischen radiologischem und funktionellem Ergebnis. Unfallchirurg 2006; 109: 837-844

8 Schneiders W, Rehberg S, Rammelt S et al. Stellenwert der Kirschner-Draht-Osteosynthese in der Behandlung der distalen Radiusfraktur. Unfallchirurg 2007; 110: 902-906

9 Young BT, Rayan GM. Outcome following nonoperative treatment of displaced distal radius fractures in low-demand patients older than 60 years. J Hand Surg [Am] 2000; 25: 19-28

10 Trumble TE, Schmitt SR, Vedder NB. Factors affecting functional outcomes of displaced intraarticular distal radius fractures. J Hand Surg [Am] 1994; 19: 325-340

\section{Priv.-Doz. Dr. med. Wolfgang Schneiders \\ Oberarzt}

Prof. Dr. med. Stefan Rammelt Oberarzt

Prof. Dr. med. Hans Zwipp

Klinikdirektor

Klinik und Poliklinik für Unfall- und Wiederherstellungschirurgie Universitätsklinikum „Carl Gustav Carus“ der TU Dresden

Fetscherstraße 74

01307 Dresden

\section{Wolfgang.Schneiders@} uniklinikum-dresden.de 